\title{
RESEARCH
}

Open Access

\section{HSPB7 regulates osteogenic differentiation of human adipose derived stem cells via ERK signaling pathway}

Chanyuan Jin(1), Ting Shuai and Zhihui Tang ${ }^{*}$

\begin{abstract}
Background: Heat shock protein B7 (HSPB7), which belongs to small heat shock protein family, has been reported to be involved in diverse biological processes and diseases. However, whether HSPB7 regulates osteogenic differentiation of human adipose derived stem cells (hASCs) remains unexplored.

Methods: The expression level of HSPB7 during the osteogenesis of hASCs was examined by quantitative reverse transcription polymerase chain reaction (qRT-PCR) and Western blot analysis. Lentivirus transfection was used to knock down or overexpress HSPB7, which enabled us to investigate the effect of HSPB7 on osteogenic differentiation of hASCs. U0126 and extracellular signal-regulated protein kinase 1/2 (ERK1/2) siRNA were used to identify the mechanism of the HSPB7/ERK1/2 axis in regulating osteogenic differentiation of hASCs. Moreover, ectopic bone formation in nude mice and osteoporosis mice model was used to investigate the effect of HSPB7 on osteogenesis in vivo.

Results: In this study, we found the expression of HSPB7 was significantly downregulated during the osteogenic differentiation of hASCs. HSPB7 knockdown remarkably promoted osteogenic differentiation of hASCs, while HSPB7 overexpression suppressed osteogenic differentiation of hASCs both in vitro and in vivo. Moreover, we discovered that the enhancing effect of HSPB7 knockdown on osteogenic differentiation was related to the activation of extracellular signal-regulated protein kinase (ERK) signaling pathway. Inhibition of ERK signaling pathway with U0126 or silencing ERK1/2 effectively blocked the stimulation of osteogenic differentiation induced by HSPB7 knockdown. Additionally, we found that HSPB7 expression was markedly increased in mouse bone marrow mesenchymal stem cells (mBMSCs) from the osteoporotic mice which suggested that HSPB7 might be utilized as a potential target in the development of effective therapeutic strategies to treat osteoporosis and other bone diseases.
\end{abstract}

Conclusion: Taken together, these findings uncover a previously unrecognized function of HSPB7 in regulating osteogenic differentiation of hASCs, partly via the ERK signaling pathway.

Keywords: Osteogenesis, Bone formation, Heat shock protein, CVHSP

\footnotetext{
* Correspondence: tang_zhihui@live.cn

Second Clinical Division, Peking University School and Hospital of

Stomatology, Beijing 100081, China
}

C C The Author(s). 2020 Open Access This article is licensed under a Creative Commons Attribution 4.0 International License, which permits use, sharing, adaptation, distribution and reproduction in any medium or format, as long as you give appropriate credit to the original author(s) and the source, provide a link to the Creative Commons licence, and indicate if changes were made. The images or other third party material in this article are included in the article's Creative Commons licence, unless indicated otherwise in a credit line to the material. If material is not included in the article's Creative Commons licence and your intended use is not permitted by statutory regulation or exceeds the permitted use, you will need to obtain permission directly from the copyright holder. To view a copy of this licence, visit http://creativecommons.org/licenses/by/4.0/ The Creative Commons Public Domain Dedication waiver (http://creativecommons.org/publicdomain/zero/1.0/) applies to the data made available in this article, unless otherwise stated in a credit line to the data. 


\section{Introduction}

Human adipose derived stem cells (hASCs) are multipotential mesenchymal stem cells (MSCs) that have the ability to differentiate into different lineages such as chondrocytes, adipocytes, and osteocytes [1]. As a type of MSCs, hASCs offer new therapeutic solutions for bone defects and metabolic diseases due to their abundant sources, ease of acquisition, low immunogenicity, and osteogenic differentiation capacity [2, 3]. However, the underlying mechanisms by which hASCs undergo osteogenic differentiation are not yet fully elucidated, thereby hindering their potential clinical applications.

It is well known that osteogenic differentiation is orchestrated by a large number of signaling pathways, such as the Wnt/ $\beta$-catenin signaling pathway, phosphatidylinositol-3kinases (PI3K)/protein serine threonine kinase (AKT) signaling pathway, transforming growth factor $\beta$ (TGF $\beta$ ) /bone morphogenetic protein (BMP) signaling pathway, and mitogen-activated protein kinase (MAPK) signaling pathway [4-7]. Among them, the extracellular signal-regulated kinase (ERK)/MAPK signaling pathway plays important roles in driving the commitment of MSCs into osteogenic lineage [8]. Researchers have revealed that ERK/MAPK could strongly activate the osteogenic-related transcription regulators such as runt-related transcription factor 2 (RUNX2) and osterix (OSX) [9]. Inactivation of ERK/MAPK signaling pathway in osteoprogenitors results in severe osteopenia and cleidocranial dysplasia [10]. Fibroblast growth factor receptor 1 (FGFR1), a transmembrane receptor which transduces extracellular signals to multiple intracellular downstream pathways, is indispensable for skeletal development via regulating osteoblast growth and differentiation [11]. Accumulating evidence has demonstrated that FGFR1 exerts its function mainly through the activation of ERK/MAPK signaling [12].

Heat shock protein B7 (HSPB7), also known as cardiovascular heat shock protein ( $\mathrm{cvHsp}$ ), belongs to the small heat shock protein family with essential roles in maintaining cellular homeostasis in a variety of both physiological and pathophysiological processes [13-15]. HSPB7 is highly expressed in the cardiac tissue and plays a critical role in cardiac metabolism [16]. The dysfunction of $H S P B 7$ is strongly associated with heart failure, dilated cardiomyopathy, and idiopathic cardiomyopathy [17]. HSPB7 has also been demonstrated to function as a tumor suppressor in multiple malignancies such as renal cell carcinoma [18, 19]. However, the effects of HSPB7 on osteogenic differentiation still remain unclear.

In this study, we evaluated the function of HSPB7 in the osteogenic differentiation of hASCs both in vitro and in vivo. We found HSPB7 knockdown promoted osteogenic differentiation of hASCs via activation of the ERK signaling pathway. These findings could contribute to better understanding of the molecular mechanisms underlying osteogenic differentiation and provide new therapeutic target for treating bone diseases.

\section{Material and methods Cell culture}

Primary human adipose derived stem cells (hASCs), human bone marrow mesenchymal stem cells (hBMSCs), and mouse MC3T3-E1 cells were purchased from ScienCell (Carlsbad, CA, USA). Human osteosarcoma U2OS cells were obtained from China Infrastructure of Cell Line Resource (Beijing, China). Cells were cultured using a proliferation medium (PM) consisting of Dulbecco's modified Eagle's medium (DMEM) (Gibco, Grand Island, NY, USA), 10\% fetal bovine serum (Gibco), and 1\% penicillin/streptomycin (Gibco). For osteogenic differentiation, hASCs and hBMSCs were cultured in osteogenic medium (OM) containing standard PM supplemented with 0.2 $\mathrm{mM}$ ascorbic acid (Sigma, St. Louis, MO, USA), $10 \mathrm{mM} \beta$ glycerophosphate (Sigma), and $100 \mathrm{nM}$ dexamethasone (Sigma). All experiments were repeated at least three times.

\section{Lentiviral transfection}

In order to overexpress $H S P B 7$, the lentivirus expressing HSPB7 and the scramble negative control (NC) were purchased from Cyagen Company (Guangzhou, China). For $H S P B 7$ knockdown, recombinant lentiviruses targeting HSPB7 (shHSPB7-1 and shHSPB7-2) and the nontargeting negative control (shNC) were purchased from GenePharma Co. (Shanghai, China). The shRNA sequences were as follows: shNC, TTCTCCGAACGTGT CACGT; shHSPB7-1, ACAGAACCUCUUCCACCUUTT; and $\operatorname{sh} H S P B 7-2$, GAACACCUUCGCUCACAAGTT. For virus transfection, cells were exposed to the lentiviral supernatant with the addition of polybrene $(5 \mu \mathrm{g} / \mathrm{mL}$, Sigma-Aldrich) for $24 \mathrm{~h}$. After $72 \mathrm{~h}$, antibiotic selection was conducted by adding puromycin $(5 \mu \mathrm{g} / \mathrm{mL}$, SigmaAldrich) to transfected cells.

\section{Small interfering RNA}

Small interfering RNAs (siRNAs) targeting ERK1/2 (siERK1, siERK2) and the negative control siRNA (siNC) were purchased from Integrated Biotech Solutions Co. (Ibsbio Co., Shanghai, China). The sequences were as follows: siNC, UUCUCCGAACGUGUCACGUTT; siERK1, GACCGGAUGUUAACCUUUAUU; and SiERK2, CAGG GUUCCUGACAGAAUAUU. To knock down ERK1/2, cells were transfected with a mixture of siERK1 and siERK2 using the Lipofectamine 3000 transfection reagent (Invitrogen, Carlsbad, CA, USA) following the manufacturer's instructions. 


\section{Alkaline phosphatase (ALP) staining}

Cells were cultured in PM or OM for 7 days. After that, cells were washed with PBS, fixed in $4 \%$ paraformaldehyde for $15 \mathrm{~min}$, incubated with the ALP substrate solution (CoWin Biotech, Beijing, China), and recorded by a scanner (Image Scanner III, GE Healthcare Bio-Sciences Corp., Piscataway, NJ, USA).

\section{Quantification of ALP activity}

Cells were washed with PBS, treated with $1 \%$ Triton X100 (Sigma), and assayed with the ALP Activity Kit (Biovision, Milpitas, CA). ALP activity was quantified by reading the absorbance at $520 \mathrm{~nm}$ and normalized to the total protein content. Total protein concentration was determined using a BCA protein assay kit (Pierce Thermo Scientific, Waltham, MA, USA).

\section{Alizarin Red S (ARS) staining and quantification}

At 14 days after osteogenic induction, cells were washed three times with PBS, fixed with $4 \%$ paraformaldehyde for $15 \mathrm{~min}$, and rinsed with Milli-Q water. Next, the calcium deposition was stained with $1 \%$ Alizarin Red S solution ( $\mathrm{pH} 4.2$, Sigma) for $20 \mathrm{~min}$. For quantification, the stain was dissolved in cetylpyridinium chloride (Sigma) and measured with a spectrophotometer at $562 \mathrm{~nm}$.

\section{Oil Red O staining}

To induce adipocyte differentiation, hASCs were cultured in adipocyte differentiation medium (AM) consisting of standard PM supplemented with $50 \mathrm{nM}$ insulin (Sigma), $0.5 \mathrm{mM}$ 3-isobutyl-1-methylxanthine (Sigma), $100 \mathrm{nM}$ dexamethasone (Sigma), and $200 \mathrm{mM}$ indomethacin (Sigma). At day 14 after adipogenic induction, cells were washed twice with PBS, fixed with $10 \%$ formalin for $30 \mathrm{~min}$, and stained with Oil Red O working solution $(0.3 \%$, Sigma). Then, the stained cells were washed with distilled water and recorded by a microscope.

\section{Immunofluorescence staining}

Cells grown on the glass coverslips were washed with ice-cold PBS, fixed with $4 \%$ formaldehyde, permeabilized with $0.2 \%$ Triton X-100, and then blocked with $1 \%$ bovine serum albumin. Next, cells were incubated with primary antibody against $\mathrm{OCN}$ (Abcam) at $4{ }^{\circ} \mathrm{C}$ overnight and then incubated with a fluorescence conjugated secondary antibody for $60 \mathrm{~min}$ at room temperature. After washing with PBS, cells were stained with DAPI for detection of nuclei. Images were visualized using a confocal microscope (Carl Zeiss, Oberkochen, Germany).

\section{RNA isolation and quantitative reverse transcription} polymerase chain reaction (qRT-PCR) analysis Total RNA was extracted using the TRIzol Reagent (Invitrogen) following the manufacturer's instructions.
The reverse transcription was performed with the PrimeScript RT Reagent Kit (Takara, Tokyo, Japan). qRTPCR was implemented with SYBR Green Master Mix (Roche Applied Science, Mannheim, Germany) on a 7500 Real-Time PCR Detection system (Applied Biosystems, Foster City, CA, USA) with gene-specific primers. The relative expression levels were measured by comparative cycle threshold (CT) method.

The primer sequences used are as follows: HSPB7, forward (F) ACTTCTCACCTGAAGACATCATTG, reverse (R) CATGACAGTGCCGTCAGC; glyceraldehyde 3-phosphatedehydrogenase (GAPDH, internal control), (F) GGTCACCAGGGCTGCTTTTA, (R) GGATCTCG CTCCTGGAAGATG; $A L P$, (F) ATGGGATGGGTGTC TCCACA, (R) CCACGAAGGGGAACTTGTC; RUNX2, (F) CCGCCTCAGTGATTTAGGGC, (R) GGGTCTGTAA TCTGACTCTGTCC; osteocalcin $(O C N)$, (F) CACTCCTC GCCCTATTGGC, (R) CCCTCCTGCTTGGACACAAAG; FGFR1, (F) CTCATCTCCTGCATGGTGGG, (R) CTGGAGTCAGCAGACACTGTT; PPAR $\gamma$, (F) GAGGAG CCTAAGGTAAGGAG, (R)GTCATTTCGTTAAAGGC TGA; BMPRIA, (F) TTCCCTGGGGTCCGGACTTA, (R) CTGCTTTCTTACGACTCCTCCA; BMPRIB, (F) TCTA TGCACACAAGGGCAAAC, (R) TGGTGGTGGCATTT ACAACG; BMPRII, (F) TGCAGCCATAAGCGAGGTTG, (R) CCCTCAAGTTCACAGCTCCT; IGF1R, (F) ACGA GTGGAGAAATCTGCGG, (R) ATGTGGAGGTAGCC CTCGAT; mouse Gapdh, (F) GGGTTCCTATAAAT ACGGACTGC, (R) TACGGCCAAATCCGTTCACA; and mouse Hspb7, (F) CGGGCTGAGAAGCTGGCA, (R) GTTGGGTCCACATCCTCTGG.

\section{Protein extraction and Western blot analysis}

Total cellular protein extracts were obtained with radioimmunoprecipitation assay (RIPA) lysis buffer supplemented with 1\% PMSF (Sigma) and 1\% phosphatase inhibitor (Roche Applied Science). The concentration of protein was measured by the BCA protein assay kit (Thermo). Next, equal amounts of samples were separated by $10 \%$ sodium dodecyl sulfate polyacrylamide gel electrophoresis and transferred to polyvinylidene fluoride (PVDF) membranes (Millipore, Billerica, MA, USA). After being blocked with skimmed milk in TBST buffer (0.1 M Tris, $150 \mathrm{mM} \mathrm{NaCl}$, and $0.1 \%$ Tween-20), the membranes were incubated overnight at $4{ }^{\circ} \mathrm{C}$ with primary antibodies against HSPB7 (Abcam, Cambridge, UK), RUNX2 (Abcam), OCN (Abcam), ERK1/2 (Cell Signaling Technology, Beverly, MA, USA), phosphorylated-ERK1/2 (Thr202/Tyr204) (Cell Signaling Technology), and GAPD $\mathrm{H}$ (HuaxingBio Science, Beijing, China). Subsequently, the membranes were washed three times with TBST and incubated with horseradish peroxidase (HRP) conjugated secondary antibodies for $1 \mathrm{~h}$ at room temperature. The bands were visualized via the ECL Western Blot Kit 
(CoWin Biotech). The intensity of bands was quantified using ImageJ analysis software (http://rsb.info.nih.gov/ij/) and normalized to GAPDH.

\section{Ectopic bone formation in vivo}

All animal protocols were performed in accordance with the laboratory animal care and use guidelines and approved by the Peking University Animal Care and Use Committee. Thirty BALB/c homozygous nude (nu/nu) male mice (8 weeks, 18-20 g) purchased from Vital Co. (Beijing, China) were randomly divided into five groups ( $n=6$ per group). Cells were induced in OM for 7 days, resuspended, incubated with Bio-Oss Collagen (Geistlich, GEWO GmbH, Baden-Baden, Germany) for $1 \mathrm{~h}$ at $37^{\circ} \mathrm{C}$, and then implanted into the dorsal subcutaneous sites of nude mice. After 8 weeks, the implants were harvested and fixed with $4 \%$ formalin for $24 \mathrm{~h}$, decalcified in $10 \%$ EDTA for 14 days, and followed by embedding in paraffin. Sections $(5 \mu \mathrm{m}$ thick) were cut and stained with hematoxylin and eosin (HE) and Masson's trichrome.

\section{Establishment of ovariectomized mouse model}

Eight-week-old female BALB/c mice weighting $20 \pm 2 \mathrm{~g}$ were obtained from Vital Co. After 1 week of acclimatization, the mice were randomly allocated to two groups: sham operation group (SHAM) and ovariectomized group (OVX). The ovariectomy was performed as described previously [20]. Twelve weeks following the ovariectomy surgery, femurs were collected and evaluated using a micro-computed tomography (micro-CT) system. The scanner was set at a current of $220 \mu \mathrm{A}$, a voltage of $60 \mathrm{kV}$, and a resolution of $9.088 \mu \mathrm{m}$ per pixel. Inveon Research Workplace (Inveon, Siemens, Munich, Germany) was used to calculate morphometric parameters, including bone volume/total volume (BV/TV), trabecular separation (Tb.Sp), and trabecular number (Tb.N) in the trabecular region (1 to $2 \mathrm{~mm}$ distal to the proximal epiphysis). Then, the femoral specimens were decalcified in $10 \%$ EDTA solution, embedded in paraffin, and stained with hematoxylin and eosin (HE). mBMSCs were collected from the tibias of SHAM and OVX mice as described previously [21].

\section{Statistical analysis}

All data were expressed as mean \pm standard deviation (SD). The data were calculated with SPSS statistics version 16.0 (SPSS Inc., Chicago, IL, USA). Independent sample $t$ test was used for comparison of two groups, and one-way analysis of variance (ANOVA) was applied for the comparison among multiple groups. A value of $P<0.05$ was considered statistically significant.

\section{Results}

HSPB7 was downregulated during osteogenic differentiation of hASCs

In order to determine the effect of HSPB7 in the process of osteogenesis, we first detected the expression level of HSPB7 during osteogenic differentiation of hASCs. qRT-PCR analysis showed the mRNA expression of HSPB7 was remarkably decreased during osteogenesis, while the expression levels of osteogenic markers RUNX2, ALP, and $O C N$ were significantly upregulated (Fig. 1a-d). Consistently, Western blot revealed a similar trend in protein expression (Fig. 1e, f).

Moreover, we compared the expression of HSPB7 among hASCs, hBMSCs, and human osteoblast-like U2OS cells. Results showed that HSPB7 was expressed at a much lower level in U2OS cells compared with hASCs and hBMSCs (SFig. 1A). Meanwhile, we compared the expression level of $H s p b 7$ between mBMSCs and mouse MC3T3-E1 cells and found that the level of $\mathrm{Hspb} 7$ was very low in mouse MC3T3-E1 cells compared with mBMSCs (SFig. 1B). These results indicated that HSPB7 might be associated with the stage of osteogenic differentiation. Additionally, we found long-term expansion of hASCs in vitro affected the expression of HSPB7. As shown in SFig. 1C, hASCs at late passages have a higher expression of $H S P B 7 \mathrm{com}$ pared with hASCs at early passages.

\section{HSPB7 overexpression inhibited osteogenic differentiation of hASCs}

To clarify whether $H S P B 7$ regulates osteogenic differentiation of hASCs, we overexpressed HSPB7 in hASCs by lentivirus transfection. Fluorescent staining revealed the transfection efficiency was more than 90\% (SFig. 2A). Western blot and qRT-PCR showed the expression level of $H S P B 7$ increased by more than 15 folds in HSPB7 overexpression hASCs in comparison to the negative control hASCs (NC) (SFig. 2B-D). After culturing the infected hASCs in OM for 7 days, ALP activity was inhibited in HSPB7 overexpression cells compared to NC cells, as revealed by ALP staining and quantitative ALP activity assay (Fig. 2a, b). Similarly, extracellular matrix mineralization was also decreased in the HSPB7 overexpression group, as determined by ARS staining and quantification on day 14 (Fig. 2c, d). Furthermore, qRT-PCR was performed to detect the mRNA expression levels of osteogenic markers, which showed that HSPB7 overexpression downregulated the expression of $R U N X 2, A L P$, and $O C N$ after 14 days of osteogenic induction (Fig. 2e-g). Additionally, Western blot and immunofluorescence showed the protein expression of OCN was reduced in the HSPB7 overexpression group (Fig. $2 \mathrm{~h}-\mathrm{j}$ ). 
A

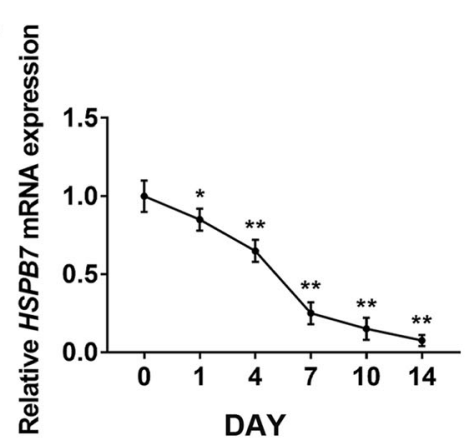

C

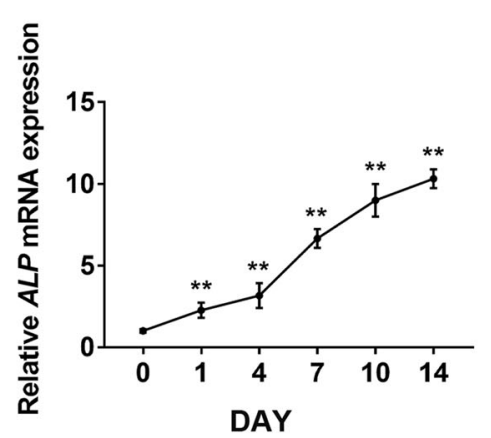

E

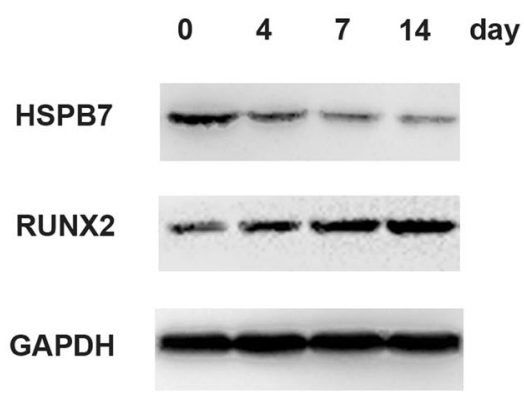

B

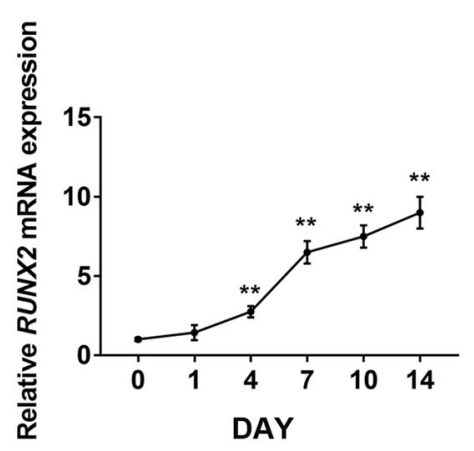

D

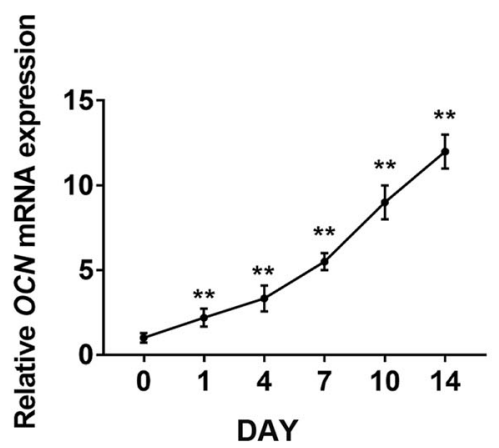

$\mathbf{F}$

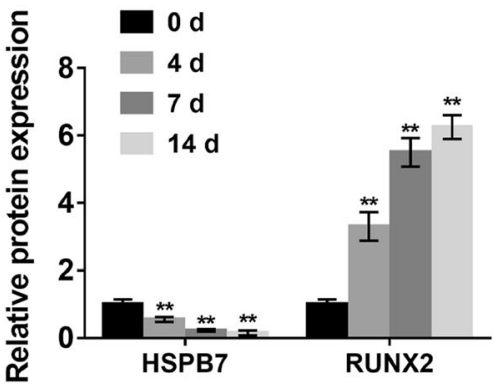

Fig. 1 HSPB7 was downregulated during the osteogenic differentiation of hASCs. a Relative mRNA expression of HSPB7 was measured by qRT-PCR at days $0,1,4,7,10$, and 14 during the osteogenic differentiation of hASCs. b-d Relative mRNA expression of RUNX2, ALP, and OCN during the osteogenic differentiation of hASCs. e After osteogenic induction, the protein expression of HSPB7 was significantly reduced, accompanied by the increased expression of RUNX2. $\mathbf{f}$ The quantitative results of e by Image J software. Glyceraldehyde 3-phosphate dehydrogenase (GAPDH) was used as a control. Data are presented as the mean \pm SD $\left({ }^{*} P<0.05\right.$, ${ }^{* *} P<$ 0.01 , compared with day 0 )

HSPB7 knockdown enhanced osteogenic differentiation of hASCs

The effect of HSPB7 on osteogenesis was further evaluated by gene knockdown experiments using shRNA. To avoid off-target effect, two different shRNA sequences against HSPB7 were employed. Following puromycin selection, the knockdown efficiency was verified by fluorescence staining (SFig. 2A), qRT-qPCR, and Western blot (SFig. 2E-G). As shown in Fig. 3a, b, ALP activity was significantly increased in the HSPB7 knockdown group compared to the shNC group. Consistently, a greater number of mineralized nodules were observed in the HSPB7 knockdown group than in the shNC group (Fig. 3c, d). Also, the mRNA expression levels of RUNX2, ALP, and OCN were increased after HSPB7 knockdown (Fig. 3e-g). Additionally, HSPB7 knockdown upregulated the protein expression of OCN (Fig. 3h-j).

To further validate the function of HSPB7 in osteogenic differentiation, the recombinant human HSPB7 protein (rhHSPB7) was utilized for rescue experiments. Results showed the addition of rhHSPB7 successfully attenuated the osteogenic differentiation capacity of HSPB7 knockdown cells as well as the shNC cells (SFig. 3A-G). In addition, we also evaluated the effect of HSPB7 on 


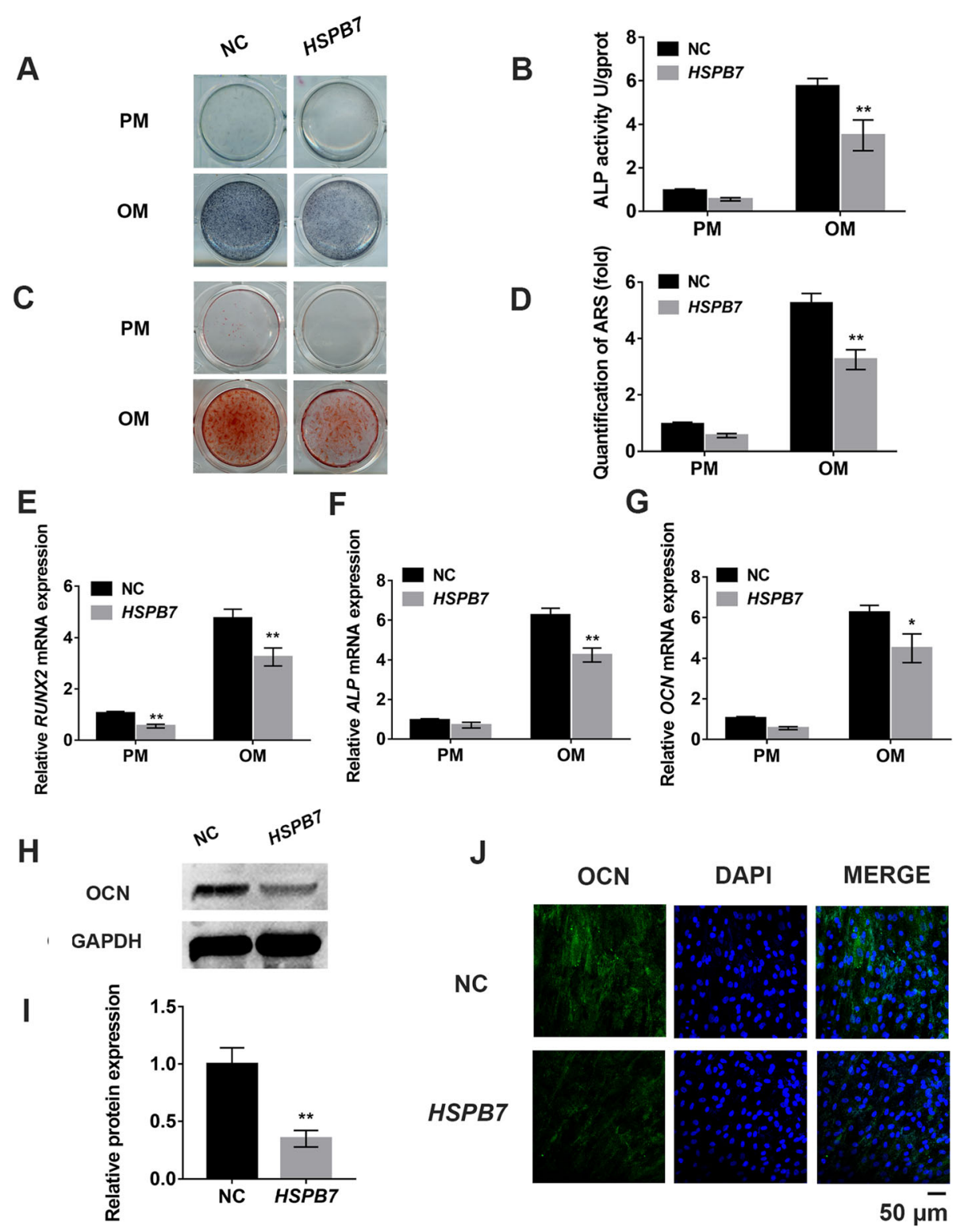

Fig. 2 HSPB7 overexpression inhibited the osteogenic differentiation of hASCs in vitro. a ALP staining in the HSPB7 overexpression (HSPB7) and control (NC) groups at day 7 of osteogenic differentiation. b Quantification of ALP activity in HSPB7 overexpression and control hASCs at day 7 of osteogenic differentiation. c Alizarin Red S staining was performed at day 14 of osteogenic differentiation. d Quantification of Alizarin Red S (ARS) staining. e- $\mathbf{g}$ Relative mRNA expression of osteogenic genes RUNX2, ALP, and OCN was assessed by qRT-PCR at day 14 of osteogenic differentiation. $\mathbf{h}, \mathbf{i}$ HSPB7 overexpression downregulated the protein expression of OCN, as determined by Western blot. $\mathbf{j}$ Confocal microscopy confirmed the downregulation of OCN protein expression in HSPB7 overexpression hASCs at day 14 of osteogenic differentiation. Scale bar = $50 \mu \mathrm{m}$. Data are presented as the mean $\pm \mathrm{SD}\left({ }^{*} P<0.05,{ }^{* *} P<0.01\right.$, compared with $\left.\mathrm{NC}\right)$

osteogenic differentiation of hBMSCs. Similarly, HSPB7 knockdown promoted osteogenesis of hBMSCs (SFig. 4AF). Collectively, these findings suggested that HSPB7 played a negative role in osteogenic differentiation.

\section{HSPB7 knockdown activated ERK1/2 signaling pathway}

To further explore the mechanism by which HSPB7 regulates osteogenic differentiation, we examined several osteogenesis-related signaling pathways and found the expression of FGFR1 and p-ERK1/2 was significantly upregulated after HSPB7 knockdown (Fig. 4a-c). By contrast, the protein level of $\mathrm{p}$-ERK1/2 was markedly decreased in HSPB7 overexpressing hASCs (SFig. 5A and $\mathrm{B})$. Due to the abovementioned results, we hypothesized that ERK signaling might be involved in HSPB7mediated osteogenic differentiation. 


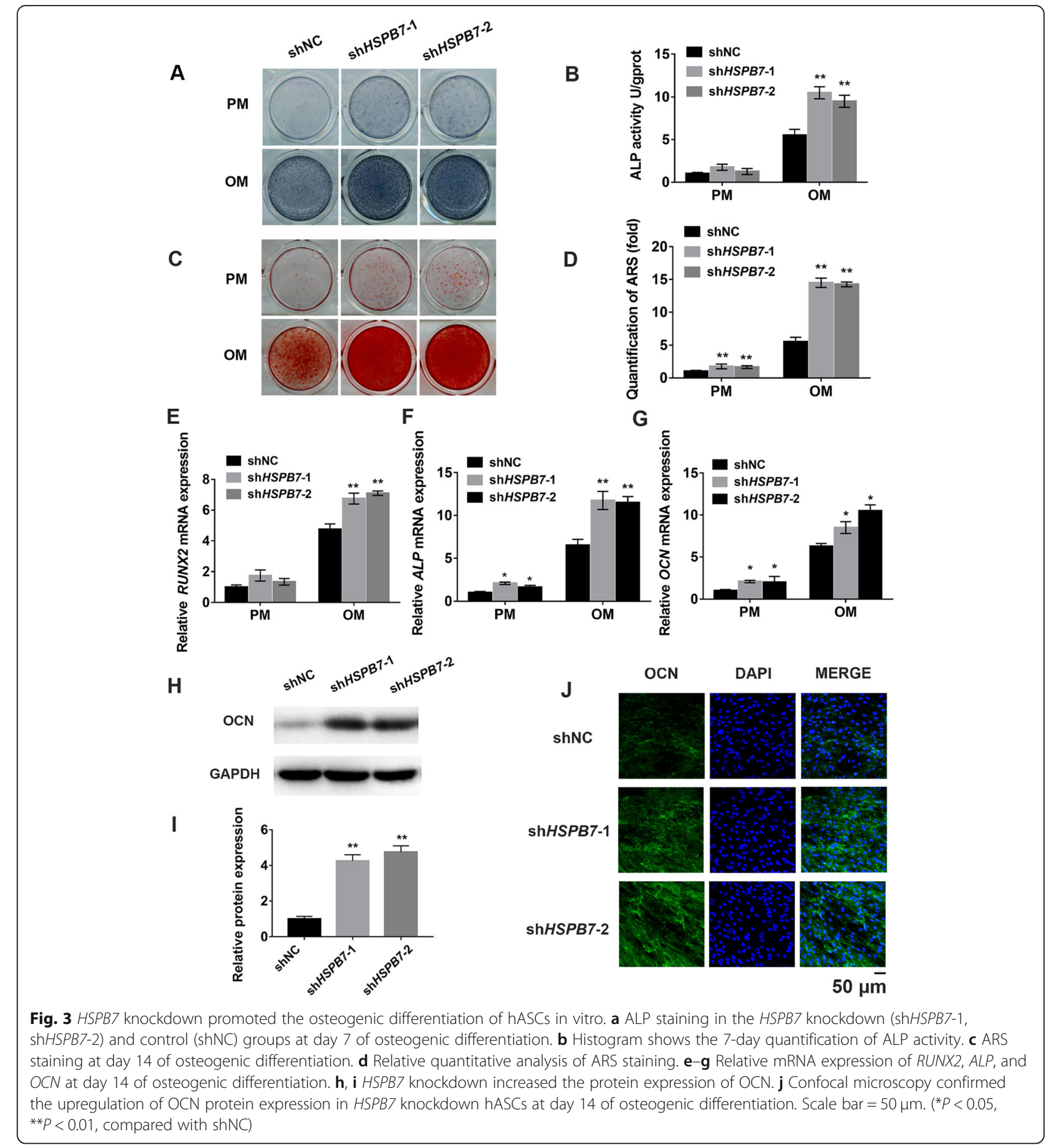

HSPB7 knockdown promoted osteogenesis through ERK1/ 2 signaling pathway

To verify whether HSPB7 regulated osteogenesis via ERK1/2 signaling pathway, we examined the inhibitory effect of ERK1/2 signaling on osteogenic differentiation in the HSPB7 knockdown group. Western blot showed the level of p-ERK1/2 was significantly inhibited with the addition of U0126 (Fig. 5a, b). Following treatment with U0126, the increased ALP activity induced by HSPB7 knockdown was effectively abrogated (Fig. 5c, d). Meanwhile, the extracellular matrix mineralization was also reduced in HSPB7 knockdown hASCs in the presence of U0126 (Fig. 5e, f). Moreover, the upregulated mRNA expression levels of osteogenic markers RUNX2, $A L P$, and $O C N$ caused by HSPB7 silencing were diminished with the addition of U0126 (Fig. $5 \mathrm{~g}-\mathrm{i}$ ). 


\section{A}

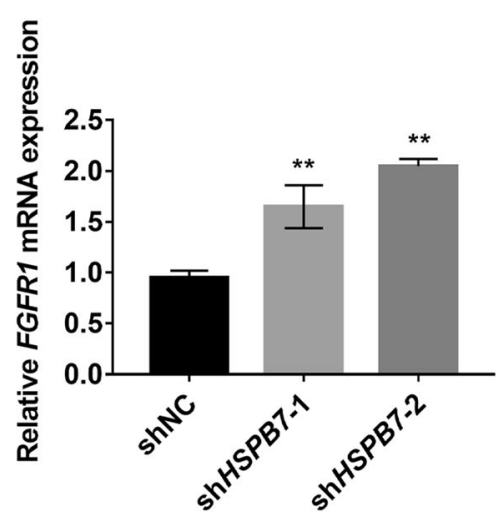

B

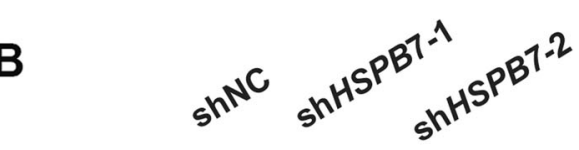

p-ERK1/2

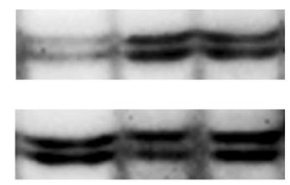

ERK1/2

GAPDH

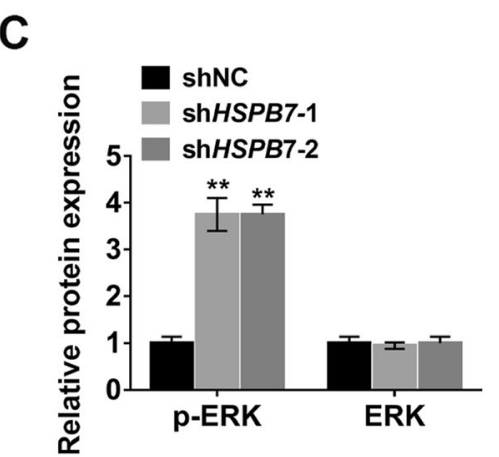

Fig. 4 HSPB7 knockdown activated ERK signaling pathway. a qRT-PCR showed HSPB7 knockdown increased the expression of FGFR1. b HSPB7 knockdown increased the level of phosphorylated ERK1/2 in hASCS. c The quantitative results of $\mathbf{b}$ by Image J software. Data are presented as the mean $\pm S D(* * P<0.01$, compared with shNC)

Next, ERK1/2 siRNA was used to confirm whether ERK1/2 knockdown could block the increased osteogenic ability induced by HSPB7 deficiency. The knockdown efficiency of ERK1/2 was demonstrated by Western blot (SFig. 6A and B). As expected, the promotive effect of HSPB7 knockdown on osteogenesis was markedly reversed in the HSPB7 and ERK1/2 double knockdown hASCs, which was revealed by ALP staining and quantification (SFig. 6C and D), ARS staining and quantification (SFig. 6E and F), and qRT-PCR (SFig. 6G-I). Taken together, these results suggested that HSPB7 regulated osteogenic differentiation through ERK signaling pathway.

Although we found HSPB7 regulated osteogenesis with an involvement of ERK signaling pathway, we cannot exclude the possibility that other signaling pathways are also involved in HSPB7-mediated osteogenic differentiation. In this study, we also found a negative correlation between the mRNA expression of HSPB7 and BMPRI/II (SFig. 7). These data suggested that BMPRI/II might be implicated in HSPB7-regulated osteogenesis. Future investigation is needed to elucidate the mechanism.

\section{HSPB7 inhibited bone formation of hASCs in vivo}

To validate our findings in vitro, we examined whether HSPB7 could affect the bone formation ability of hASCs in vivo. As shown in Fig. 6a, HE staining and Masson's trichrome staining revealed the amount of newly generated bone was higher in HSPB7 knockdown hASCs compared with the control cells. On the contrary, mice implanted with HSPB7 overexpression hASCs showed much less bone-like tissue (Fig. 6b). Furthermore, quantitative measurements using SPOT 4.0 software confirmed that the volume of newly formed bone was increased in $H S P B 7$ knockdown hASCs but reduced in HSPB7 overexpression hASCs (Fig. 6c). These results indicated that HSPB7 might suppress osteogenic differentiation of hASCs in vivo.

\section{HSPB7 promoted adipogenesis of hASCs}

It has been reported that osteogenesis and adipogenesis of MSCs are competing and reciprocal [22]. Thus, we also investigated the role of $H S P B 7$ in adipogenesis. We observed that HSPB7 was upregulated during adipogenic differentiation of hASCs (SFig. 8A). Oil Red O staining and qRTPCR showed that HSPB7 overexpression significantly promoted adipogenic differentiation of hASCs (SFig. 8B-D).

\section{HSPB7 was increased in OVX mice}

The imbalance between osteogenesis and adipogenesis is associated with various bone-related diseases, such as 


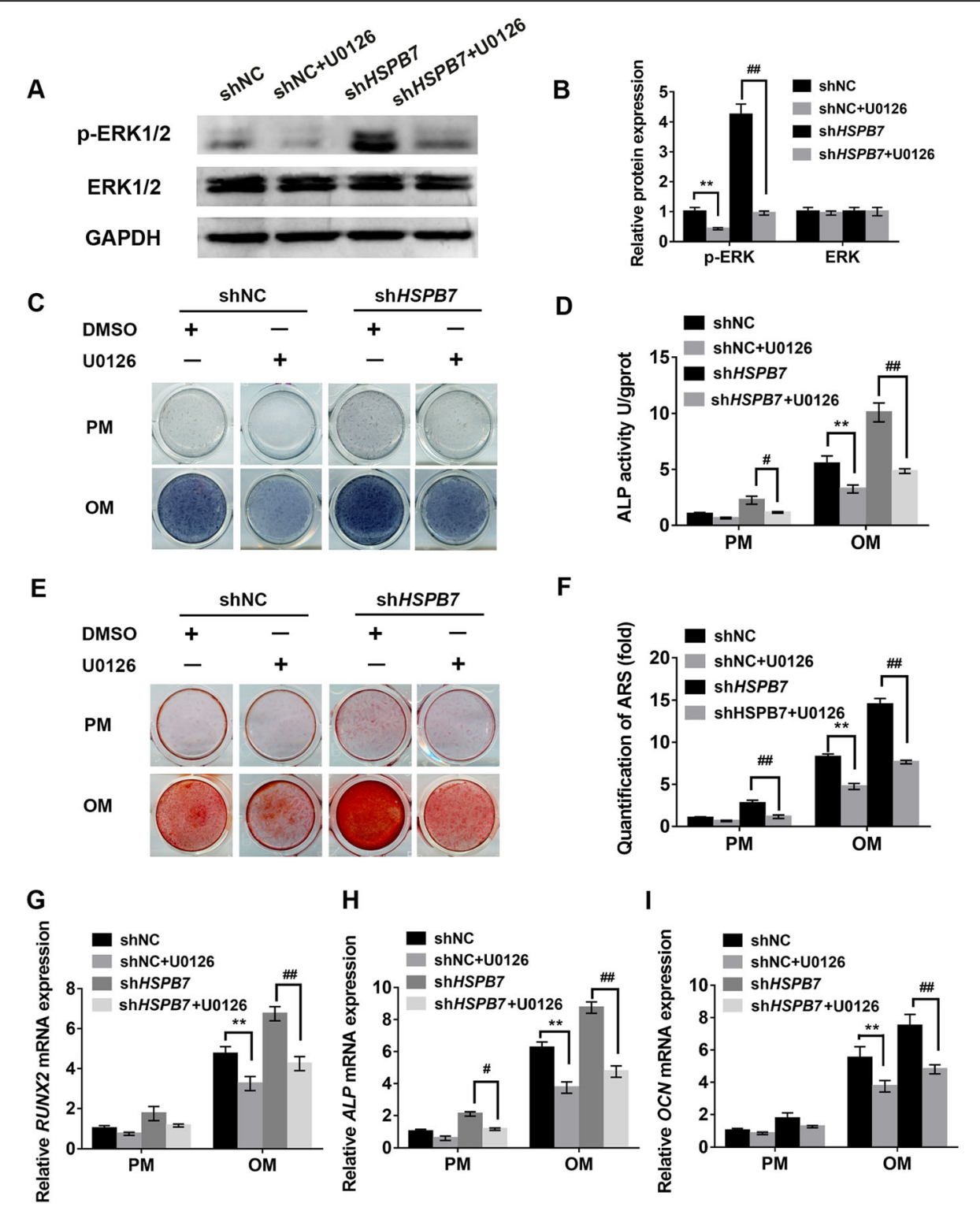

Fig. 5 Inhibiting ERK signaling pathway with U0126 reversed the enhancing effect of HSPB7 knockdown on osteogenesis of hASCS. a U0126 reduced the level of phosphorylated ERK1/2 in ShNC and ShHSPB7 hASCs. $\mathbf{b}$ The quantitative results of $\mathbf{a}$ by Image J software. c ALP staining at day 7 of osteogenic differentiation. U0126 (10 $\mu \mathrm{M})$ or DMSO was incubated for 7 days. d Histogram shows the 7-day quantification of ALP activity. e ARS staining in ShNC, shHSPB7 groups treated with U0126 $(10 \mu \mathrm{M})$ or DMSO (control) for 14 days. f Relative quantitative analysis of ARS staining. g-i Relative mRNA expression of RUNX2, ALP, and OCN at day 14 of osteogenic differentiation in the presence or absence of U0126 (10 $\mu \mathrm{M})$. DMSO was used as a control. Data are presented as the mean $\pm \mathrm{SD}\left({ }^{*} P<0.05,{ }^{*} P<0.01\right.$, compared with shNC)

osteoporosis. Based on above results, it was reasonable to hypothesize that HSPB7 might be abnormally expressed in osteoporotic mice. To verify this hypothesis, we constructed osteoporotic mouse models by ovariectomy. HE staining and micro-CT showed that the trabecular number (Tb.N) and bone volume/total volume (BV/TV) were significantly reduced, whereas the trabecular separation (Tb.SP) was increased in OVX mice compared to those in SHAM mice, which confirmed the establishment of osteoporotic models (SFig. 9A-D). Then, mBMSCs were collected from OVX and SHAM mice and identified by flow cytometry. The protein expression of HSPB7 was greatly increased in mBMSCs from osteoporotic mice, as revealed by Western blot (SFig. 9E and F). These data suggested that HSPB7 might be involved in the pathogenesis of osteoporosis. More experimental studies are needed to further explore the role of HSPB7 in osteoporosis. 


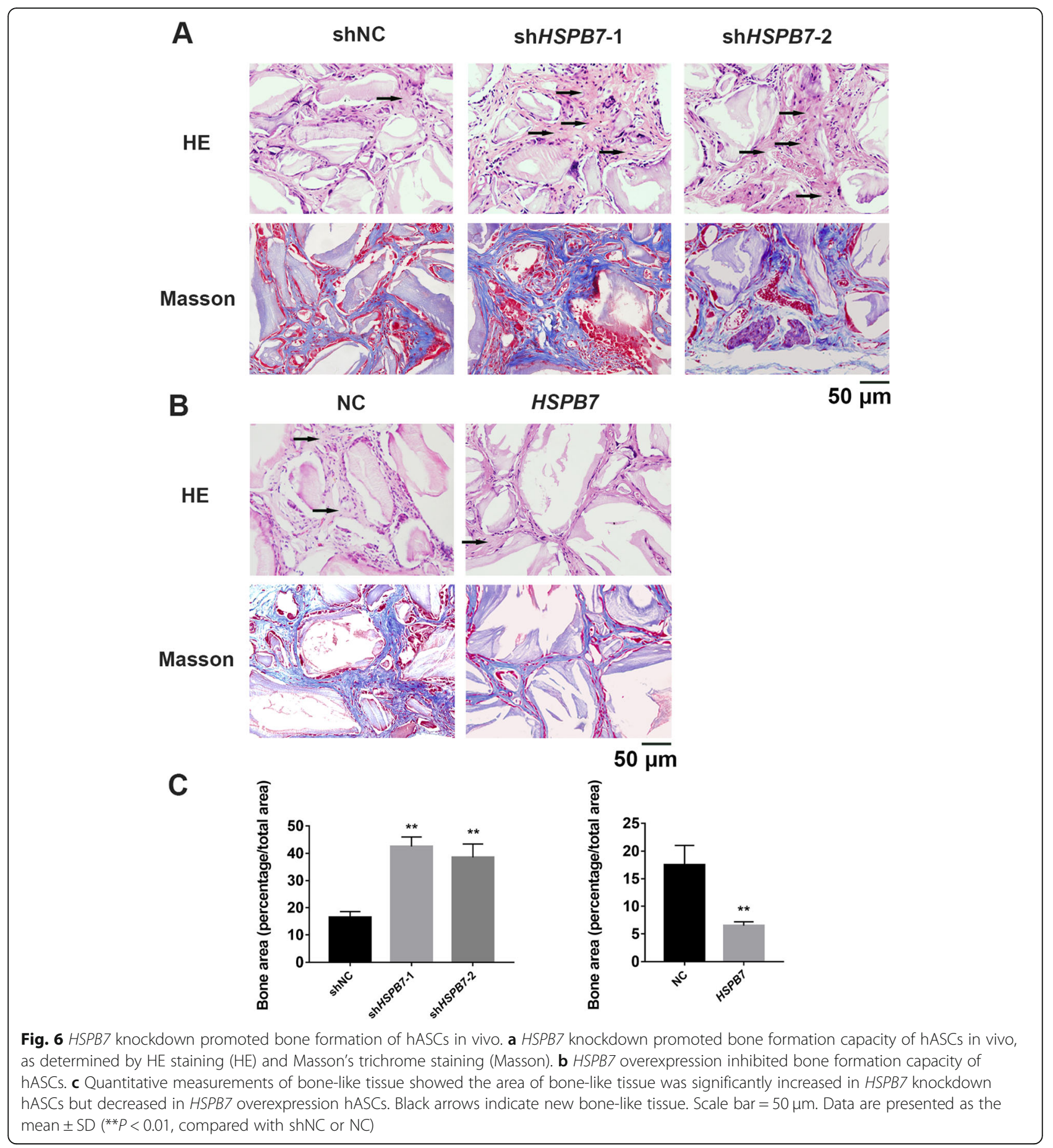

\section{Discussion}

During recent years, hASCs isolated from adipose tissue have emerged as an attractive cell source for bone tissue engineering which sheds a light on bone repair and regeneration [23-25]. However, employing hASCs to promote bone repair is far from being applied in clinical routine due to the limited knowledge about osteogenic differentiation. Thus, it is of great significance to investigate the molecular basis of osteogenesis. Herein, we demonstrated that HSPB7 was markedly reduced during osteogenesis of hASCs and HSPB7 knockdown enhanced osteogenic capacity of hASCs both in vitro and in vivo.

HSPB7, located on 1p36.13, is a member of heat shock protein (HSP) family that plays essential roles in protein folding and cellular protein homeostasis [26]. Heat shock 
proteins have been implicated in diverse range of cellular processes, such as stress response, cell proliferation, and apoptosis [27-29]. Recent studies have revealed that heat shock proteins also play crucial roles in osteogenic differentiation. Flanagan et al. reported that heat shock protein B8 (HSPB8) knockdown significantly reduced osteogenic differentiation potential of dental pulp stem cells [30]. Chen et al. showed extracellular heat shock protein 70 (HSP70) enhanced osteogenesis of human mesenchymal stem cells (hMSCs) [31]. A study by Zhang et al. demonstrated low-intensity pulsed ultrasound stimulated osteogenesis of hASCs possibly by increasing the expression of HSP7O and HSP9O [32]. $H S P B 7$, which is highly expressed in the heart, has been intensively investigated in multiple cardiac pathologies [33]. In the present study, our results provided novel insight into how HSPB7 regulated osteogenic differentiation of hASCs. We first showed that HSPB7 knockdown enhanced hASC osteogenesis through ERK signaling.

Extracellular signal-regulated kinase (ERK) pathway, also known as the Ras-Raf-MEK-ERK pathway, is involved in a variety of physiological processes such as cell proliferation, division, and survival [34, 35]. Numerous studies have revealed that ERK signaling pathway plays vital roles in the osteogenic differentiation [36-39]. With regard to the relationship between $H S P B 7$ and ERK signaling pathway, Naderi found HSPB7 overexpression in breast cancer cells reduced the level of p-ERK [18]. In agreement with the above study, we found HSPB7 overexpression reduced the level of p-ERK, while HSPB7 knockdown significantly activated ERK signaling pathway. To clarify the involvement of ERK signaling pathway in HSPB7-mediated osteogenic differentiation of hASCs, we examined whether inhibiting ERK signaling pathway with U0126 or siERK1/2 could attenuate the effect of HSPB7 knockdown on osteogenesis of hASCs. Results showed inhibition of ERK signaling pathway effectively blocked the enhancing effect of HSPB7 knockdown on osteogenesis. Although our study demonstrated that ERK signaling pathway played a crucial role in osteogenesis induced by HSPB7 knockdown, it is likely that other signaling pathways are also involved in this process. Previous studies indicated that HSPB7 was associated with diverse important signaling pathways. For example, Naderi found HSPB7 overexpression could downregulate the expression of p-AKT which is closely associated with osteogenic differentiation [18]. In this study, we observed the expression of $B M P R I / I I$ was negatively regulated by HSPB7, which suggested that BMPRI/II might be involved in HSPB7-regulated osteogenesis. Future studies are still needed to elucidate the mechanism of HSPB7-mediated osteogenesis.

Osteoporosis is a major public health problem which has a high incidence and great risk of fracture. To date, molecular pathways involved in the pathogenesis of osteoporosis remain elusive. Uncovering the mechanisms underlying osteoporosis is important for developing novel therapies and effective prevention strategies [40]. It has been well established that the imbalance between adipogenesis and osteogenesis contributes to osteoporosis [41]. In this study, we found HSPB7 expression was significantly increased during adipogenic differentiation of hASCs. We hypothesized that HSPB7 might be aberrantly expressed in osteoporosis model. Therefore, we constructed OVX mice and examined HSPB7 expression in mBMSCs from OVX. As expected, we found the protein expression of HSPB7 was significantly increased in OVX mice, which suggested the potential involvement of HSPB7 in the pathogenesis of osteoporosis. The present study provides new insight into the

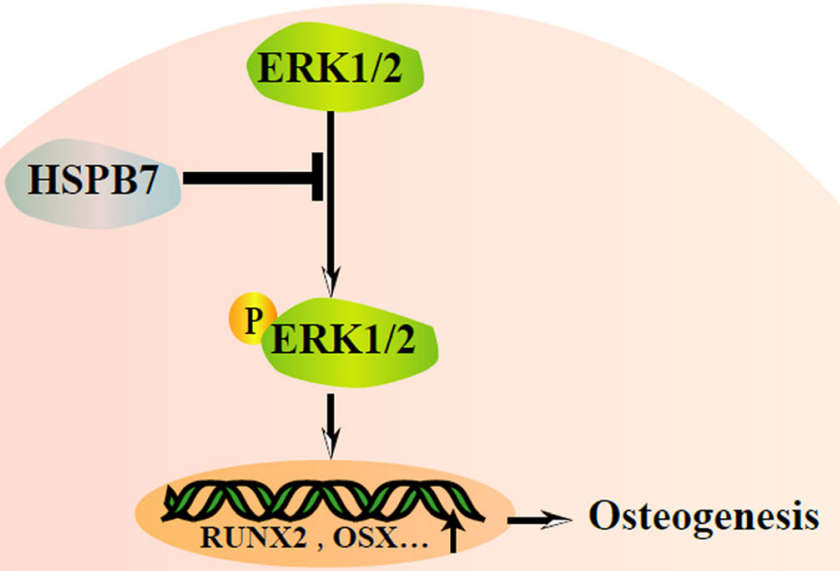

Fig. 7 Schematic diagram of the regulation of osteogenesis by HSPB7. HSPB7 regulated the osteogenic differentiation of hASCS via ERK1/2 signaling 
molecular basis of osteoporosis and implied that HSPB7 might be used as a potential diagnostic marker and therapeutic target for osteoporosis.

\section{Conclusions}

In summary, this is the first study to identify the negative role of HSPB7 in osteogenic differentiation of hASCs with an involvement of ERK signaling pathway (Fig. 7). These findings could provide not only new ideas for exploring new approaches in stimulating osteogenesis of hASCs but also a potential therapeutic target for the skeletal diseases.

\section{Supplementary information}

Supplementary information accompanies this paper at https://doi.org/10. 1186/s13287-020-01965-4.

Additional file 1: Supplementary Figure 1. The expression of HSPB7 in different cell types. (A) The mRNA expression of HSPB7 in hASCS, hBMSCs and U2OS cells. (B) The mRNA expression of Hspb7 in mBMSCs and MC3T3-E1 cells. (C) The mRNA expression level of HSPB7 was upregulated with passage in hASCs. Results are presented as the mean \pm SD. $\left({ }^{*} P<0.05,{ }^{* *} P<0.01\right)$

Additional file 2: Supplementary Figure 2. Lentiviral transfection to overexpress or knockdown HSPB7 in hASCs. (A) Images of GFP-positive hASCs under a normal microscope and a fluorescence microscope. Scale bar $=200 \mu \mathrm{m}$. (B) Relative mRNA expression of HSPB7 in NC, HSPB7 groups. (C, D) Protein levels of HSPB7 in the HSPB7 overexpression group (HSPBA) and negative control (NC) group. (E) Relative mRNA expression of HSPB7 in shNC, shHSPB7-1 and shHSPB7-2 groups. $(F, G)$ Protein levels of HSPB7 in the HSPB7 knockdown group (shHSPB7-1, shHSPB7-2) and negative control (shNC) group. GAPDH was used as an internal control. Results are presented as the mean $\pm S D$. ( ${ }^{*} P<0.01$, compared with $N C$ or shNC).

Additional file 3: Supplementary Figure 3. Recombinant human HSPB7 protein reversed the enhancing effect of HSPB7 knockdown on osteogenesis of hASCs. (A, B) ALP activity of shNC and shHSPB7 hASCs at day 7 in the presence or absence of recombinant human HSPB7 (rhHSPB7, $400 \mathrm{ng} / \mathrm{mll}$ ). Equal volume of PBS was used as a control. (C.D) ARS staining and quantification at day 14. (E-G) Relative mRNA levels of RUNX2, ALP and OCN determined by qRT-PCR at day 14 after osteogenic induction. Results are presented as the mean \pm SD. $\left({ }^{*} /{ }^{*} P<0.05\right.$, ${ }^{* *} /{ }^{\# \#} P<$ $0.01,{ }^{*}$ compared with shNC, ${ }^{*}$ compared with shHSPB7).

Additional file 4: Supplementary Figure 4. HSPB7 knockdown promoted osteogenesis of hBMSCs. (A) qRT-PCR showed that the mRNA expression of HSPB7 was decreased during the osteogenic differentiation of hBMSCs. (B) The knockdown efficiency of HSPB7 in hBMSCs. (C) ALP staining in HSPB7 knockdown (shHSPB7-1, shHSPB7-2) and control (shNC) groups on day 7 after osteogenic induction. (D) Relative quantitative analyses of ALP activity on day 7 after osteogenic induction. (E) ARS staining on day 14 after osteogenic induction. (F) Relative quantitative analysis of ARS staining. Results are presented as the mean \pm SD. $\left({ }^{*} P<0.05,{ }^{* *} P<\right.$ 0.01 , compared with day 0 or shNC).

Additional file 5: Supplementary Figure 5. HSPB7 overexpression inhibited ERK signalling pathway. (A) HSPB7 overexpression reduced the level of phosphorylated ERK1/2 in hASCs. (B) The quantitative results of (A) by Image J software. Data are presented as the mean $\pm \mathrm{SD}\left({ }^{* *} P<0.01\right.$, compared with $\mathrm{NC}$.

Additional file 6: Supplementary Figure 6. Knockdown of ERK1/2 blocked the promotive effect of HSPB7 knockdown on osteogenesis of hASCS. (A, B) The knockdown efficiency of ERK $1 / 2$ was verified by Western blot. (C, D) ALP activity was significantly reduced in HSPB7/ERK1/ 2 double knockdown hASCs in comparison with HSPB7 knockdown hASCs. (E, F) ARS staining and quantification at day 14 of osteogenic differentiation. (G-I) Relative mRNA expression of RUNX2, ALP and OCN at day 14 of osteogenic differentiation. Results are presented as the mean \pm SD. $\left({ }^{*} / \# P<0.05,{ }^{* *} / \# P<0.01,{ }^{*}\right.$ compared with shNC+siNC, ${ }^{\#}$ compared with shHSPB7+siNC).

Additional file 7: Supplementary Figure 7. HSPB7 regulated BMPRI/II expression. QRT-PCR analysis showed that HSPB7 knockdown significantly upregulated the mRNA expression of BMPRIA (A), BMPRIB (B) and BMPRII (C), whereas HSPB7 overexpression downregulated the expression of $B M P R I A, B M P R I B$ and BMPRII. Results are presented as the mean $\pm S D$. $\left({ }^{*} P<0.05,{ }^{*} P<0.01\right.$, compared with shNC).

Additional file 8: Supplementary Figure 8. HSPB7 regulated adipogenesis of hASCS. (A) The mRNA expression of HSPB7 was increased during the adipogenic differentiation of hASCs, as detected by qRT-PCR. (B) Lentivirus transfection was conducted to overexpress HSPB7 in hASCS and confirmed by qRT-PCR. (C) Cells were treated with proliferation medium (PM) or adipogenic medium (AM) for 14 days. HSPB7 overexpression increased the lipid accumulation of hASCs, as revealed by Oil red $\mathrm{O}$ staining. (D) HSPB7 overexpression promoted the expression of adipogenic marker gene PPARY, as determined by qRT-PCR. Results are presented as the mean $\pm \mathrm{SD}$. $\left({ }^{*} P<0.05,{ }^{*} P<0.01\right.$, compared with NC).

Additional file 9: Supplementary Figure 9. HSPB7 expression was increased in mBMSCs from OVX mice. (A) Representative HE staining and Micro CT images at 12 weeks after ovariectomy. Scale bars for HE staining and Micro CT represent $50 \mu \mathrm{m}$ and $1 \mathrm{~mm}$, respectively. (B-D) Quantitative analyses of parameters regarding bone microstructure, including trabecular number (Tb.N), trabecular bone volume/tissue volume (BV/TV), and trabecular spacing (Tb.Sp). (E) Western blot analysis showed that HSPB7 expression was significantly increased in mBMSCs from OVX mice compared with SHAM mice. (F) The band intensities of (E) were analyzed by Image J software. GAPDH was used as the internal control. Results are presented as the mean $\pm S D$. ( ${ }^{* *} P<0.01$, compared with SHAM group).

\section{Abbreviations}

hASCs: Human adipose derived stem cells; hBMSCs: Human bone marrow mesenchymal stem cells; mBMSCs: Mouse bone marrow mesenchymal stem cells; PM: Proliferation medium; OM: Osteogenic medium; AM: Adipogenic medium; siRNA: Small interfering RNA; NC: Negative control; HE: Hematoxylin and eosin; ALP: Alkaline phosphatase; ARS: Alizarin Red S; qRTPCR: Quantitative reverse transcription polymerase chain reaction; GAPD H: Glyceraldehyde 3-phosphatedehydrogenase; OCN: Osteocalcin; RUNX2: Runt-related transcription factor 2; PBS: Phosphate-buffered saline; TBST: Tris-buffered saline Tween-20; $\mathrm{p}$-ERK1/2: Phosphorylated extracellular signal-regulated kinase1/2; rhHSPB7: Recombinant human HSPB7 protein

\section{Acknowledgements}

This study was supported by the National Science Foundation of China (81800942) and Chinese Postdoctoral Science Foundation (2018 M631442).

\section{Authors' contributions}

C.J. performed the experiments, collected the data, analyzed the data, and wrote the paper. T.S. performed the experiments and collected the data. Z.T. designed the research and supervised the work. All authors approved the final version of the manuscript.

\section{Availability of data and materials}

The authors confirm that all data underlying the findings are fully available.

\section{Ethics approval and consent to participate}

This study was performed in strict accordance with the recommendations of the Institutional Animal Care and Use Committee of the Peking University Health Science Center.

\section{Consent for publication}

Not applicable.

\section{Competing interests}

The authors indicate no potential conflicts of interest. 
Received: 25 April 2020 Accepted: 5 October 2020

Published online: 23 October 2020

\section{References}

1. Liu G, Chen X. Isolating and characterizing adipose-derived stem cells. Methods Mol Biol. 1842;2018:193-201.

2. Caetano G, Wang W, Murashima A, Passarini JR Jr, Bagne L, Leite M, et al. Tissue constructs with human adipose-derived mesenchymal stem cells to treat bone defects in rats. Materials (Basel). 2019;12(14):2268.

3. Tajima S, Tobita M, Mizuno H. Current status of bone regeneration using adipose-derived stem cells. Histol Histopathol. 2018;33(7):619-27.

4. Maeda K, Kobayashi Y, Koide M, Uehara S, Okamoto M, Ishihara A, et al. The regulation of bone metabolism and disorders by Wnt signaling. Int J Mol Sci. 2019;20(22):5525.

5. Jiang H-T, Ran C-C, Liao Y-P, Zhu J-H, Wang H, Deng R, et al. IGF-1 reverses the osteogenic inhibitory effect of dexamethasone on BMP9-induced osteogenic differentiation in mouse embryonic fibroblasts via PI3K AKT/ COX-2 pathway. J Steroid Biochem Mol Biol. 2019;191:105363.

6. Zhang P, Zhang H, Lin J, Xiao T, Xu R, Fu Y, et al. Insulin impedes osteogenesis of BMSCs by inhibiting autophagy and promoting premature senescence via the TGF- $\beta 1$ pathway. Aging (Albany NY). 2020;12(3):2084-100

7. Wu M, Chen G, Li Y-P. TGF- $\beta$ and BMP signaling in osteoblast, skeletal development, and bone formation, homeostasis and disease. Bone Res. 2016;4:16009.

8. Lu N, Malemud CJ. Extracellular signal-regulated kinase: a regulator of cell growth, inflammation, chondrocyte and bone cell receptor-mediated gene expression. Int J Mol Sci. 2019;20(15):3792.

9. Franceschi RT, Ge C. Control of the osteoblast lineage by mitogen-activated protein kinase signaling. Curr Mol Biol Rep. 2017;3(2):122-32.

10. Kim J-M, Yang Y-S, Park KH, Oh H, Greenblatt MB, Shim J-H. The ERK MAPK pathway is essential for skeletal development and homeostasis. Int J Mol Sci. 2019;20(8):1803.

11. Sargar KM, Singh AK, Kao SC. Imaging of skeletal disorders caused by fibroblast growth factor receptor gene mutations. Radiographics. 2017;37(6): 1813-30.

12. Zhang W, Xue D, Hu D, Xie T, Tao Y, Zhu T, et al. Secreted klotho protein attenuates osteogenic differentiation of human bone marrow mesenchymal stem cells in vitro via inactivation of the FGFR1/ERK signaling pathway. Growth Factors. 2015;33(5-6):356-65.

13. Mercer EJ, Lin Y-F, Cohen-Gould L, Evans T. Hspb7 is a cardioprotective chaperone facilitating sarcomeric proteostasis. Dev Biol. 2018;435(1):41-55.

14. Wu T, Mu Y, Bogomolovas J, Fang X, Veevers J, Nowak RB, et al. HSPB7 is indispensable for heart development by modulating actin filament assembly. Proc Natl Acad Sci U S A. 2017;114(45):11956-61.

15. Wu D, Vonk JJ, Salles F, Vonk D, Haslbeck M, Melki R, et al. The N terminus of the small heat shock protein HSPB7 drives its polyQ aggregationsuppressing activity. J Biol Chem. 2019;294(25):9985-94.

16. Rüdebusch J, Benkner A, Poesch A, Dörr M, Völker U, Grube K, et al. Dynamic adaptation of myocardial proteome during heart failure development. PLoS One. 2017:12(10):e0185915.

17. Chen FF, Xia YL, Xu CQ, Li SS, Zhao YY, Wang XJ, et al. Common variant rs7597774 in ADD2 is associated with dilated cardiomyopathy in Chinese Han population. Int J Clin Exp Med. 2015;8(1):1188-96.

18. Naderi A. SRARP and HSPB7 are epigenetically regulated gene pairs that function as tumor suppressors and predict clinical outcome in malignancies. Mol Oncol. 2018;12(5):724-55.

19. Lin J, Deng Z, Tanikawa C, Shuin T, Miki T, Matsuda K, et al. Downregulation of the tumor suppressor HSPB7, involved in the p53 pathway, in renal cell carcinoma by hypermethylation. Int J Oncol. 2014;44(5):1490-8.

20. Jin C, Jia L, Tang Z, Zheng Y. Long non-coding RNA MIR22HG promotes osteogenic differentiation of bone marrow mesenchymal stem cells via PTEN/ AKT pathway. Cell Death Dis. 2020;11(7):601.

21. Jin C, Zhang P, Zhang M, Zhang X, LV L, Liu H, et al. Inhibition of SLC7A11 by sulfasalazine enhances osteogenic differentiation of mesenchymal stem cells by modulating BMP2/4 expression and suppresses bone loss in ovariectomized mice. J Bone Miner Res. 2017;32(3):508-21.

22. Chen $Q$, Shou P, Zheng C, Jiang M, Cao G, Yang Q, et al. Fate decision of mesenchymal stem cells: adipocytes or osteoblasts? Cell Death Differ. 2016; 23(7):1128-39.

23. Wu V, Helder MN, Bravenboer N, Ten Bruggenkate CM, Jin J, Klein-Nulend J, et al. Bone tissue regeneration in the oral and maxillofacial region: a review on the application of stem cells and new strategies to improve vascularization. Stem Cells Int. 2019;2019:6279721.

24. Hutchings $G$, Moncrieff $L$, Dompe $C$, Janowicz K, Sibiak R, Bryja A, et al. Bone regeneration, reconstruction and use of osteogenic cells; from basic knowledge, animal models to clinical trials. J Clin Med. 2020;9(1):139.

25. Mohiuddin OA, Campbell B, Poche JN, Ma M, Rogers E, Gaupp D, et al. Decellularized adipose tissue hydrogel promotes bone regeneration in criticalsized mouse femoral defect model. Front Bioeng Biotechnol. 2019;7:211.

26. Jacob P, Hirt H, Bendahmane A. The heat-shock protein/chaperone network and multiple stress resistance. Plant Biotechnol J. 2017;15(4):405-14.

27. Zininga T, Ramatsui L, Shonhai A. Heat shock proteins as immunomodulants. Molecules. 2018;23(11):2846

28. Malitan HS, Cohen AM, MacRae TH. Knockdown of the small heat-shock protein p26 by RNA interference modifies the diapause proteome of Artemia franciscana. Biochem Cell Biol. 2019:97(4):471-9.

29. Izumi M. Heat shock proteins support refolding and shredding of misfolded proteins. Plant Physiol. 2019;180(4):1777-8.

30. Flanagan M, Li C, Dietrich MA, Richard M, Yao S. Downregulation of heat shock protein B8 decreases osteogenic differentiation potential of dental pulp stem cells during in vitro proliferation. Cell Prolif. 2018;51(2):e12420.

31. Chen E, Xue D, Zhang W, Lin F, Pan Z. Extracellular heat shock protein 70 promotes osteogenesis of human mesenchymal stem cells through activation of the ERK signaling pathway. FEBS Lett. 2015;589(24 Pt B):4088-96.

32. Zhang Z, Ma Y, Guo S, He Y, Bai G, Zhang W. Low-intensity pulsed ultrasound stimulation facilitates in vitro osteogenic differentiation of human adipose-derived stem cells via up-regulation of heat shock protein (HSP)70, HSP90, and bone morphogenetic protein (BMP) signaling pathway. Biosci Rep. 2018;38(3):BSR20180087.

33. Liao W-C, Juo L-Y, Shih Y-L, Chen Y-H, Yan Y-T. HSPB7 prevents cardiac conduction system defect through maintaining intercalated disc integrity. PLoS Genet. 2017;13(8):e1006984.

34. Degirmenci $U$, Wang M, Hu J. Targeting aberrant RAS/RAF/MEK/ERK signaling for cancer therapy. Cells. 2020;9(1):198.

35. Olea-Flores M, Zuñiga-Eulogio MD, Mendoza-Catalán MA, Rodríguez-Ruiz HA, Castañeda-Saucedo E, Ortuño-Pineda C, et al. Extracellular-signal regulated kinase: a central molecule driving epithelial-mesenchymal transition in cancer. Int J Mol Sci. 2019;20(12):2885.

36. Wang C-X, Ge X-Y, Wang M-Y, Ma T, Zhang Y, Lin Y. Dopamine D1 receptormediated activation of the ERK signaling pathway is involved in the osteogenic differentiation of bone mesenchymal stem cells. Stem Cell Res Ther. 2020;11(1):12.

37. Ni L, Kuang Z, Gong Z, Xue D, Zheng Q. Dihydroartemisinin promotes the osteogenesis of human mesenchymal stem cells via the ERK and Wnt/ßcatenin signaling pathways. Biomed Res Int. 2019;2019:3456719.

38. Huang Y, Lin Y, Rong M, Liu W, He J, Zhou L. 20(S)-hydroxycholesterol and simvastatin synergistically enhance osteogenic differentiation of marrow stromal cells and bone regeneration by initiation of Raf/MEK/ERK signaling. J Mater Sci Mater Med. 2019;30(8):87.

39. Zheng G, Xie Z, Wang P, Li J, Li M, Cen S, et al. Enhanced osteogenic differentiation of mesenchymal stem cells in ankylosing spondylitis: a study based on a three-dimensional biomimetic environment. Cell Death Dis. 2019;10(5):350.

40. Föger-Samwald U, Dovjak P, Azizi-Semrad U, Kerschan-Schindl K, Pietschmann P. Osteoporosis: pathophysiology and therapeutic options. EXCLI J. 2020;19:1017-37.

41. Pierce $J L$, Begun DL, Westendorf JJ, McGee-Lawrence ME. Defining osteoblast and adipocyte lineages in the bone marrow. Bone. 2019:118:2-7.

\section{Publisher's Note}

Springer Nature remains neutral with regard to jurisdictional claims in published maps and institutional affiliations. 